\title{
Estado da arte sobre o cuidar em neonatologia: compromisso da enfermagem com a humanização na unidade de terapia intensiva neonatal
}

Este estudo teve por objetivo caracterizar a produção científica brasileira de Enfermagem sobre neonatologia e a humanização do cuidado aos recém-nascidos no período entre 2000 e 2011. A partir de uma revisão de literatura integrativa, foram selecionados 13 artigos, os quais constituíram a amostra do estudo. Os resultados evidenciaram diversidade temática, destacando-se o tema Humanização no cuidado de Enfermagem. Os sujeitos mais frequentemente abordados foram os profissionais de equipes de saúde e os enfermeiros. A maioria dos autores apresentou propostas para a solução de problemas identificados. Contudo, ainda são necessários mais estudos sobre a temática.

Descritores: Neonatologia, Humanização, Cuidado, Enfermagem.

\section{State-of-the-art study on the care in neonatology: commitment of nursing with humanization in the neonatal intensive care unit}

This study aimed to characterize the Brazilian scientific production of Nursing about Neonatology and the humanization in the care to newborns from 2000 to 2011 . From a review of integrative literature, 13 articles were selected, which constituted the sample for the study. The results evidenced theme diversity, with theme Humanization in Nursing care standing out. The most frequently subjects approached were the professionals from health teams and nurses. The greater part of the authors presented proposals for solving the identified problems. However, further studies on this theme are still necessary. Descriptors: Neonatology, Humanization, Care, Nursing.

Estado del arte sobre el cuidar en neonatología: compromiso de la enfermería con la humanización en la unidad de terapia intensiva neonatal Este estudio tuvo como objetivo caracterizar la producción científica brasileña de Enfermería sobre neonatología y la humanización del cuidado a los recién nacidos en el período entre el 2000 y el 2011. A partir de una revisión de literatura integrativa, se seleccionaron 13 artículos, los cuales constituyeron la muestra del estudio. Los resultados pusieron en evidencia diversidad temática, destacándose el tema Humanización en el cuidado de Enfermería. Los sujetos más frecuentemente abordados fueron los profesionales de equipos de salud y los enfermeros. La mayoría de los autores presentó propuestas para la solución de problemas identificados. Sin embargo, aún son necesarios más estudios sobre la temática.

Descriptores: Neonatología, Humanización, Cuidado, Enfermería.

\section{INTRODUÇÃO}

E ste estudo tem por objetivo caracterizar a produção científica brasileira de Enfermagem sobre neonatologia e a humanização do cuidado aos recém-nascidos no - período entre 2000 e 2011.

Refletir sobre a natureza do cuidado é essencial para a enfermagem como disciplina e profissão. Contudo, qualquer reflexão desse teor deve levar em conta o ser e o meio em que está inserido, pois o cuidado não se dá no vazio, mas no contexto das experiências humanas, onde a subjetividade, a consciência, a vida e a espiritualidade estão presentes.

No âmbito neonatal, o cuidar torna-se um grande desafio para o enfermeiro, em decorrência de o mesmo tornar-se amplo e complexo, exigindo uma nova postura do profissional de saúde, permitindo-o assim manter relações sentimentais, empáticas e de compaixão com o outro(1). Não esquecendo o respeito às individualidades, a segurança do recém-nato e o acolhimento ao bebê e sua família.

\section{REVISÃO DA LITERATURA}

O período neonatal compreende os primeiros 28 dias de vida do bebê. O recém-nascido a termo é aquele cuja idade gestacional é de 37 a 42 semanas e o pré-termo, (RNPT) todo aquele que tem menos de 37 semanas $^{(2,3)}$.

A literatura nacional evidencia que o recém-nascido pré-termo e/ou recém-nascido de muito baixo peso $(<1500 \mathrm{~g})$ são os mais atingidos pela morbimortalidade no período neonatal ${ }^{(4-6)}$.

A prática do cuidado a esses bebês deve ser estruturada e organizada no sentido de atender a uma população sujeita a 
riscos ${ }^{(3)}$. Dessa maneira, o cuidado humanizado é "o resultado de um processo no qual se conjugam, de forma bastante estreita, sentimentos, valores, atitudes e princípios científicos com o objetivo de satisfazer os indivíduos nele envolvidos"(7).

E, com a inclusão da família no processo assistencial nas UTINs, existe a necessidade de instrumentalizar os profissionais com "conhecimentos em psicologia familiar, apego entre mãe e filho, relacionamento interpessoal e direitos humanos, para que a atuação seja pautada no modelo de atenção à saúde humanizada"(8).

\section{METODOLOGIA}

Neste artigo, utilizou-se a revisão de literatura integrativa que permite "a síntese do estado do conhecimento de um determinado assunto", possibilitando conclusões gerais"(9-11).

As etapas da pesquisa seguiram-se desta maneira: Identificação do tema e seleção da questão norteadora; Estabelecimento dos critérios para inclusão e exclusão de estudos: amostragem ou busca na literatura, onde foram selecionadas os artigos publicados em banco de dados científicos na Literatura Latino-Americana e do Caribe em Ciências da Saúde (LILACS), Scientific Electronic Library Online (SciELO), Revista Brasileira de Enfermagem REBEn, artigos na íntegra que retratassem a temática e publicações em língua portuguesa nos últimos onze anos.

O refinamento resultou em 13 artigos que serviram como fonte de consulta, sendo um sobre Ações em enfermagem na UTIN; três sobre Revisão da literatura sobre Enfermagem e Neonatologia; cinco sobre Humanização do cuidado em Enfermagem na UTIN e quatro sobre Inserção e orientação da família no cuidado ao recém-nascido na UTIN. Os dados literários foram selecionados, codificados e categorizados da seguinte forma: Processo de trabalho em enfermagem em Neonatologia - Humanização da assistência na UTIN - A participação da família no cuidado ao recém-nascido em UTIN. Como antepenúltima etapa, realizouse a avaliação dos estudos incluídos na revisão integrativa. $\mathrm{Na}$ penúltima etapa, os resultados foram interpretados e, na última, elaborou-se a revisão e a síntese do conhecimento.

A análise dos dados foi realizada através da identificação dos dados de localização do artigo, ano e periódico de publicação, autoria, objetivo, metodologia, resultados principais; além da sintetização por similaridade de conteúdo.

\section{RESULTADOS E DISCUSSÃO}

Dos 13 artigos científicos (Quadro 01) selecionados pelos critérios de inclusão previamente estabelecidos, dois foram na EERP/USP, um da FEN/UFG, um encontrado na base de dados LILACS, um na REBEn e oito na base da SciELO.

Dentre as publicações analisadas, todas são de periódicos nacionais, dez de revisão e três são recortes de teses de doutorado. Interessante destacar que não foi mencionado nenhum livro ou capítulo de livro publicado sobre a temática.

Da síntese por similaridade de conteúdo foi possível evidenciar algumas temáticas dos discursos dos autores, tais como: Processo de trabalho em enfermagem em Neonatologia - Humanização da assistência na UTIN - A participação da família no cuidado ao recém-nascido em UTIN. Estas, por sua vez, serão desmembradas a seguir.

\section{Processo de trabalho em enfermagem e o cuidado em Neonatologia}

O trabalho da enfermagem "tem um caráter subsidiário, complementar e o mesmo objeto de trabalho, que é o corpo humano individual e coletivo"(8).

Nas últimas décadas, a ciência e a tecnologia vêm avançando no campo da saúde, com destaque para a Neonatologia, que tem progredido com o surgimento de novos equipamentos, terapêuticas e conhecimentos ${ }^{(4)}$. Isso ocorre principalmente nas UTINs, ambientes onde são utilizados técnicas e procedimentos sofisticados, que podem propiciar condições para a reversão dos distúrbios que colocam em risco a vida dos bebês de alto risco ${ }^{(12-17)}$.

Esta, embora indiscutivelmente necessária quando se associa a tecnologia às UTIs, por vezes cria uma barreira muito grande entre o que é humano e o que é artificial ${ }^{(18)}$, desviando o foco da atenção que deveria estar no paciente ${ }^{(6)}$.

A tecnologia como fundamento do cuidado neonatal requer um repensar de todas as formas de relacionamento entre bebês, profissionais e família, na adequação de sua utilização a diversos saberes, oferecendo cuidado individualizado, seguro, ético e humano(18).

Dessa maneira, a UTIN apresenta-se como um espaço de trabalho que exige maiores conhecimentos e habilidades do profissional de enfermagem ${ }^{(4)}$.

A evolução do saber/fazer da enfermagem em UTIN é considerada como algo importante ${ }^{(13)}$. Pois os profissionais precisam "organizar seu espaço e suas tarefas entre os bebês e os equipamentos. Daí a necessidade de uma equipe multidisciplinar, especializada, capaz de prestar atendimento indiviadualizado ao bebê e à família" $(4,8)$.

O trabalho do enfermeiro é indispensável, pois ele une o conhecimento científico à realidade e à prática da UTIN. Com efeito, ele pode reconhecer as necessidades do bebê e planejar sua assistência, consciente do compromisso com o ser humano global, dotado de sensibilidade, necessitando de carinho, atenção e amor ${ }^{(13)}$.

\section{Humanização da assistência na UTIN}

Há de se considerar que, dentre as profissões da saúde, a enfermagem é a que está mais próxima do paciente ${ }^{(13)}$. A ela cabe relacionar-se com o sujeito, havendo valorização do cuidado ao paciente, manifestando sua presença ativa como um todo(6).

Ao considerar a possibilidade da prestação de um cuidado humanizado, percebe-se uma interação estabelecida entre o profissional de enfermagem e o $\mathrm{RN}$, além deste constituir um dos seus componentes, revestindo-se de extrema relevância, por tratar-se de um fator responsável pelo seu desenvolvimento saudável ${ }^{(17)}$.

A palavra humanização pode ser entendida como a maneira de ver e considerar o ser humano a partir de uma visão geral, buscando superar a fragmentação da assistência ${ }^{(12,13)}$. 


\begin{tabular}{|c|c|c|}
\hline Procedência & Título & Autor(es) \\
\hline SciELO & A dimensão humana do cuidado em enfermagem & Silva AL \\
\hline SciELO & $\begin{array}{l}\text { O cuidar de crianças egressas da unidade de terapia intensiva neonatal no } \\
\text { espaço domiciliar: instrumentos mediadores das famílias }\end{array}$ & Moraes JRMM, Cabral IE \\
\hline LILACS & $\begin{array}{l}\text { O significado de cuidar da família na UTI neonatal: crenças da equipe de } \\
\text { enfermagem }\end{array}$ & Pedroso GER, Bousso RS \\
\hline SciELO & $\begin{array}{l}\text { A experiência vivida pelas famílias de crianças hospitalizadas em uma } \\
\text { Unidade de Terapia Intensiva Neonatal }\end{array}$ & $\begin{array}{l}\text { Centa ML, Moreira EC, Pinto } \\
\text { MNGHR }\end{array}$ \\
\hline EERP/USP & $\begin{array}{l}\text { Análise da teoria humanística e a relação interpessoal do } \\
\text { enfermeiro no cuidado ao recém-nascido }\end{array}$ & $\begin{array}{l}\text { Rolim KMC, Pagliuca LMF, Cardoso } \\
\text { MVLML }\end{array}$ \\
\hline EERP/USP & $\begin{array}{l}\text { O discurso e a prática do cuidado ao recém-nascido de risco: } \\
\text { refletindo sobre a atenção humanizada }\end{array}$ & Rolim KMC, Cardoso MVLML \\
\hline SciELO & $\begin{array}{l}\text { O processo de trabalho da equipe de enfermagem na UTI neonatal } \\
\text { e o cuidar humanizado }\end{array}$ & Oliveira BRG et al. \\
\hline FEN/UFG & Humanização do Cuidado da UTI Neonatal & Reichert APS, Lins RNP, Collet N \\
\hline SciELO & Enfermagem ou Maternagem: Interação Enfermeira - Recém Nascido & Falcão Júnior JSP et al. \\
\hline SciELO & $\begin{array}{l}\text { Promoção de vínculo afetivo na Unidade de Terapia Intensiva } \\
\text { Neonatal: um desafio para as enfermeiras }\end{array}$ & Conz CA, Merighi MAB, Jesus MCP \\
\hline SciELO & $\begin{array}{l}\text { Produção de conhecimento sobre o cuidado ao recém-nascido em UTI } \\
\text { Neonatal: contribuição da enfermagem brasileira }\end{array}$ & Costa R, Padilha MI, Monticelli M \\
\hline SciELO & Tecnologia como fundamento do cuidar em neonatologia & Sá Neto JA, Rodrigues BMRD \\
\hline REBEn & $\begin{array}{l}\text { Enfermagem em Unidade de Terapia Intensiva Neonatal: perfil da produção } \\
\text { científica brasileira }\end{array}$ & Cruz ATCT et al. \\
\hline
\end{tabular}

Quadro 1 - Distribuição dos estudos segundo caracterização da procedência, título, autores, periódico, ano, e considerações temáticas. 


\section{Periódico}

Acta Paulista de Enfermagem

Texto \& Contexto em Enfermagem

Acta Scientiarum. Health Sciences

Texto \& Contexto em Enfermagem

Revista Latino-Americana de Enfermagem

Revista Latino-Americana de Enfermagem

Texto \& Contexto em Enfermagem

Revista Eletrônica de Enfermagem

Esc Anna Nery R Enferm

Revista da Escola de Enfermagem da USP

Revista da Escola de Enfermagem da USP

Texto \& Contexto em Enfermagem

Cogitare Enfermagem
Trata sobre a evolução histórica da tecnologia em Neonatologia, enfatizando aspectos conceituais e reflexivos sobre o impacto da tecnologia no processo do cuidar.

\section{Considerações Temáticas}

Argumenta sobre a necessidade do cuidado de si como fundamental para o cuidado humano na enfermagem.

Apresenta os instrumentos mediadores que as famílias utilizam no domicílio para cuidar de criança egressa da terapia intensiva neonatal, descrevendo e analisando como se deu sua internalização e incorporação pelas famílias no cotidiano do cuidar.

Elucida o significado de cuidar da família para os membros da equipe de enfermagem da UTI neonatal, identifica como esse significado se reflete na prática e conhece as crenças que norteiam o cuidado da família em UTI neonatal.

Identificar a experiência vivida pelos familiares de crianças internadas em Unidade de Terapia Intensiva Neonatal em um hospital de Curitiba.

Reflete sobre a utilidade na prática de conceitos da Teoria Humanística de Enfermagem, numa dissertação de mestrado, utilizando como referência o modelo de análise de teorias de Meleis.

Identifica a opinião da enfermeira acerca da humanização do cuidado ao récem-nascido (RN) de risco na Unidade de Terapia Intensiva Neonatal (UTIN) e a seus familiares, utilizando como referencial a Teoria de Paterson e Zderad (1976).

Diagnostica o processo de trabalho de enfermagem na Unidade de Terapia Intensiva Neonatal; identifica a percepção da equipe sobre seu objeto de trabalho, quais são os instrumentos de trabalho utilizados, qual é a finalidade do trabalho, qual é o produto final obtido com o trabalho e que ações são desenvolvidas para a humanização da assistência de enfermagem.

Identifica ações de enfermagem, descritas na literatura, que contribuem para a humanização da assistência na Unidade de Terapia Intensiva Neonatal (UTIN).

Correlaciona o comportamento afetivo e interacional das enfermeiras durante a oferta de alimento ao recém-nascido por meio da técnica do copinho, em uma unidade neonatal, a um cuidado essencial de enfermagem, tendo em vista a vulnerabilidade dessa clientela.

Investiga a vivência das enfermeiras no processo de vínculo afetivo no cuidado entre recém-nascidos internados em UTIN e seus pais.

Reflete sobre o estado da arte na Enfermagem brasileira acerca do cuidado ao recém-nascido em UTI Neonatal.

Caracteriza a produção científica brasileira de Enfermagem sobre Neonatologia e Recém-nascidos de risco no período entre 2004 e 2007. 
A enfermagem brasileira muito tem contribuído em pesquisas na seara da humanização do cuidado, tendo em vista as especificidades de seu objeto de trabalho. Especificamente na UTIN, a humanização da assistência de enfermagem procura pautar-se no cuidado singular ao RN e sua família, na integralidade e no respeito à vida ${ }^{(8)}$.

\section{A participação da família no cuidado ao recém-nascido em UTIN}

É importante, portanto, que os profissionais que atuam em UTIN estejam aptos para amenizar o dano emocional causado aos familiares pela internação da criança, por meio de uma assistência humanizada prestada não só à criança, mas também aos pais. Agindo de forma integral, tentando interagir com os familiares, atendendo necessidades, apoiando, ensinando e incentivando a participação destes no cuidado(2).

Para os profissionais da área, "receber e cuidar de um prematuro de $800 \mathrm{~g}$, por exemplo, em uma UTIN, não é mais um mistério; mas o é para a família, seja no espaço hospitalar ou domiciliar" (15).

No decorrer da hospitalização do neonato, a enfermeira pode se aproximar da família, desenvolvendo uma abordagem educativa na perspectiva mais dialógica do que monológica(15), apoiando e promovendo condições para que os pais possam ver e tocar seu bebê, proporcionando um ambiente acolhedor ${ }^{(13,16)}$.

Entretanto, compreender a família não é um processo simples. Pois, durante a internação dos bebês, "[...] o profissional enfermeiro deve procurar conhecê-las e compreender reações, sentimentos, signos, significados, hábitos, costumes e valores. Para isso, faz-se necessário interagir, apoiar e orientar, amenizando a dor"(2).

Dessa maneira, o enfermeiro da UTINs pode criar possibilidades que permitam ampliar o foco do cuidado, em busca da perspectiva de se ver a criança internada e a família como um conjunto a ser cuidado(7).

\section{CONSIDERAÇÕES FINAIS}

Acredita-se que a atenção à humanização do cuidado neonatal é um elemento necessário à reorganização das práticas de saúde.

Pode-se dizer que os artigos apresentados desenham a linha de pensamento da necessidade de investimento na formação dos profissionais das UTINs, promovendo não somente a capacitação técnica, mas também a sensibilização do enfermeiro para que planeje a assistência pautada nos fundamentos da humanização e da integralização do cuidado.

\section{Referências}

1. Silva AL. A dimensão humana do cuidado em enfermagem. Acta Paul Enferm. 2000;13(Esp):86-90.

2. Centa ML, Moreira EC, Pinto MNGHR. A experiência vivida pelas famílias de crianças hospitalizadas em uma unidade de terapia intensiva neonatal. Texto Contexto Enferm. 2004;13(3):444-51.

3. Rolim KMC, Cardoso MVLML. O discurso e a prática do cuidado ao recém-nascido de risco: refletindo sobre a atenção humanizada. Rev Latinoam Enferm. 2006;14(1):85-92.

4. Cruz ATCT, et al. Enfermagem em Unidade de Terapia Intensiva Neonatal: perfil da produção científica brasileira. Cogitare Enferm. 2011;16(1):141-47.

5. Moraes JRMM, Cabral IE. O cuidar de crianças egressas da unidade de terapia intensiva neonatal no espaço domiciliar: instrumentos mediadores das famílias. Texto Contexto Enferm. 2004;13(3):452-8.

6. Rolim KMC, Pagliuca LMF, Cardoso MVLML. Análise da teoria humanística ea relação interpessoal do enfermeiro no cuidado ao recém-nascido. Rev Latinoam Enferm. 2005;13(3).

7. Pedroso GER, Bousso RS. O significado de cuidar da família na UTI neonatal: crenças da equipe de enfermagem. Acta Sci Health Sci. 2004;26(1):129-134. 8. Oliveira BRG et al. O processo de trabalho da equipe de enfermagem na UTI neonatal e o cuidar humanizado. Texto Contexto Enferm. 2006;15(Esp):105-13. 9. Mendes KDS, Silveira RCCP, Galvão CM. Revisão integrativa: método de pesquisa para a Incorporação de evidências na saúde e na enfermagem. Texto Contexto Enferm. 2008;17(4):758-64.
10. Souza MT, Silva MD, Carvalho R. Revisão integrativa: o que é e como fazer. Einstein. 2010;8(1 Pt 1):102-6.

11. Ferreira MA, Castro ES, Mendes PW. A integração no cuidado: uma questão na enfermagem fundamental. Esc Anna Nery Rev Enferm. 2005;A.9(1):39-45.

12. Reichert APS, Lins RNP, Collet N. Humanização do cuidado da UTI neonatal [Internet]. 2007. Disponivel em: http://www.fen.ufg.br/revista/v9/n1/v9n1a16.htm 13. Rolim KMC, Cardoso MVLML. O discurso e a prática do cuidado ao recém-nascido de risco: refletindo sobre a atenção humanizada. Rev Latinoam Enferm. 2006;14(1):85-92.

14. Costa R, Padilha Ml, Monticelli M. Produção de conhecimento sobre o cuidado ao recém-nascido em UTI neonatal: contribuição da enfermagem brasileira. Rev Esc Enferm USP. 2010;44(1):199-204.

15. Moraes JRMM, Cabral IE. O cuidar de crianças egressas da unidade de terapia intensiva neonatal no espaço domiciliar: instrumentos mediadores das famílias. Texto Contexto Enferm. 2004;13(3):452-8.

16. Conz CA, Merighi MAB, Jesus MCP. Promoção de vínculo afetivo na Unidade de Terapia Intensiva Neonatal: um desafio para as enfermeiras. Rev Esc Enferm USP. 2009;43(4):849-55.

17. Biesbroeck FCC, Cardim MG, Nascimento MAL. Entre a enfermagem e a maternagem: um estudo sobre a interação enfermeira e recém-nascido. Esc Anna Nery Rev Enferm. 2007;11(1):80-85.

18. Sá Neto JA, Rodrigues BMRD. Tecnologia como fundamento do cuidar em neonatologia. Texto Contexto Enferm. 2010;19(2):372-7. 